\title{
ADAPTIVE BUSBAR DIFFERENTIAL PROTECTION BASED ON CURRENT TRANSFORMER SATURATION DEGREES
}

\author{
R. Abd Allah', S. Moussa ${ }^{2}$, E. H. Shehab-Eldin'², M. N. G. Hamed ${ }^{1}$ \\ 1) Egyptian Electricity Holding Co., El-Kureimate Power plant, Egypt. \\ 2) Helwan University, Faculty of engineering, Cairo, Egypt.
}

\begin{abstract}
This paper presents an advanced technique for avoiding current transformer saturation effects on busbar protection. The technique classifies the CT saturation into degrees depending on calculating the correlation coefficient between the saturated secondary current of the faulted circuit and the summation of the other secondary currents for the un-faulted circuits. This estimation is performed for external faults. The technique is useful in adapting the differential relay characteristics of the protected busbar to avoid false operation. The proposed technique has attractive features include the simplicity for on line implementation, not affected by the remanent flux in the CT core, and it is independent of the CT parameters/characteristics of secondary burden. The accuracy and robustness of the introduced algorithm are demonstrated through extensive simulation cases reflecting a wide range of variation in fault conditions and CT parameters.

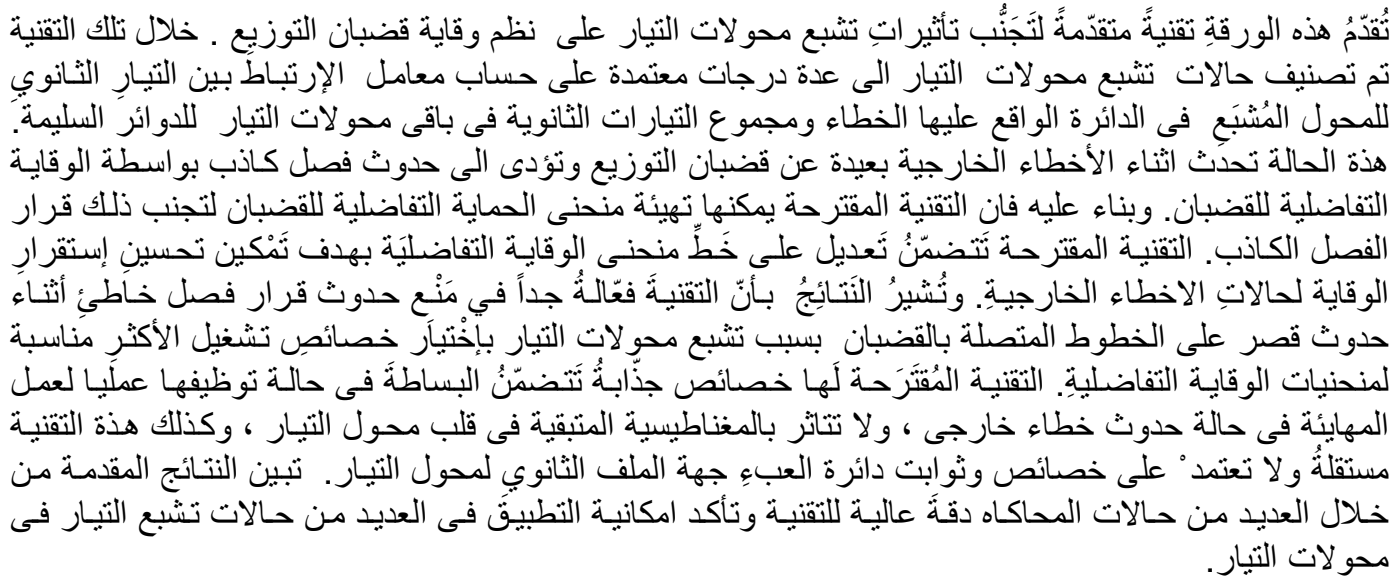

Keywords: Fault Detection, CT Saturation Detection, Saturation Effects, Time-To-Saturation, Adaptive Differential Relays

\section{INTRODUCTION}

Busbars are vital parts of power networks because they link incoming circuits connected from sources, to outgoing circuits which feed loads. The effect of a single bus fault is equivalent to many simultaneous faults

and usually, due to the concentration of supply circuits, involves high current magnitudes. Highspeed busbar protection is often required to limit the damaging effects on equipment and system stability or to maintain service to as much load as possible.

Differential protection is the most sensitive and reliable method for protecting a station buses. The phasor summation of all the measured current entering and leaving the bus must be zero unless there is a fault within the protective zone. For a fault not in the protective zone, the faulted circuit is energized at a much higher level, near CT saturation or with varying degrees of CT saturation, giving rise to possible high false differential currents.

Many methods are used to avoid the CT saturation effects. Recently, many software techniques are provided to solve these problems and each method has its advantage and disadvantage. Some techniques uses a DC component equal and opposite of that in the primary circuit generated by a circuit added to the secondary winding [1]. Other techniques used a magnetization curve and the equivalent circuit of a CT for compensating secondary current of CT during saturation. The second has practical difficulties, as it depends on CT parameters/characteristics and secondary burdens [2]. 
Also, the artificial neural networks (ANN) are used in this area. ANN attempts to learn the nonlinear characteristics of $\mathrm{CT}$ magnetization and restructures the waveform based on the learned characteristics. This method could not be applied to different CT's due to the variations of CT's saturation characteristics and the secondary burdens [3]. Some other algorithms prevent relay operation during CT saturation. This may result in longer trip times. A method for compensating the secondary current of CT's is based on the ideal proportional transient secondary fault current. A portion of measured secondary current following the fault occurrence is described using regression analysis [4]. Another method utilizes the last four consequent samples during the unsaturated portion of each cycle for solving a set of equations. By solving these equations, the constants of the primary fault current equation are obtained for reconstructing the secondary current during the saturated part each cycle. This scheme calculates the correct primary time constant by repeating the calculations of the algorithm using different values of time constant and chooses the value that gives the smallest error [5].

The application of the adaptation idea is indispensable to assure proper operation of the protection for external faults with small through currents accompanied with CT saturation due to DC component. Waldemar Rebizant, Tammam Hayder, Ludwig Schiel [6] developed An Adaptive Differential Protection Scheme which depends on calculation of the initial value (amplitude) and time constant of the decaying DC component as well as the amplitude of the current fundamental frequency component for the time instant just after fault inception. Also, they calculate the expected CT saturation period, i.e. time instants of saturation beginning and end. These estimates are performed for the signals generated from each CT of the protected element. This approach also needs the CT data and reactance and resistance of the network at the fault spot which are used for the estimation of CT saturation starting time.

Through-out this work a new technique is proposed for selecting the more suitable operating characteristics for adaptive busbar differential relays according to CT saturation degrees in order to avoid saturation effects. This technique uses the correlation concept for measuring the saturation severity of the distorted secondary current signal in case of external faults.

Before discussing our proposed adaptive busbar differential protection technique, it is useful to give a glance of the operating characteristic of one of the recent busbar differential protection.

\section{BUSBAR DIFFERENTIAL PROTECTION CHARACTERISTICS}

The Pickup characteristic of the differential protection can be set in the parameters for $I_{d}>$ (pickup value) and for the $\mathrm{k}$ factor which considers the linear and non-linear current transformer errors. See figure 1. Differential currents above the set characteristic lead to tripping [7].

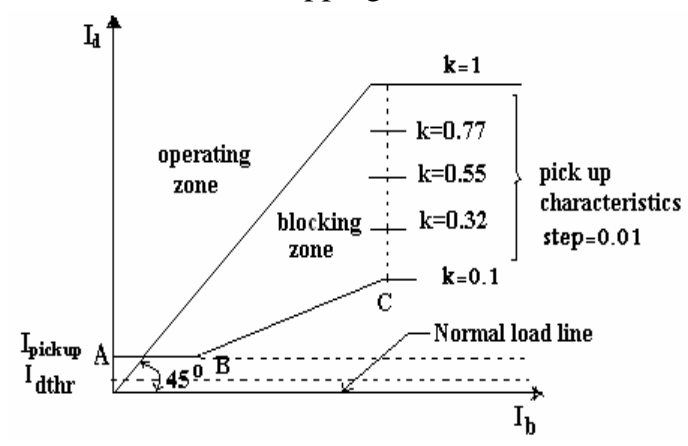

Fig. 1 Operating characteristics of percentage differential relay

The relay uses multi-slope operating characteristics; where each differential current sample, is the summation of the corresponding current samples from each $\mathrm{CT}$ in the protected zone, i.e.

$$
i_{d}(x)=\sum_{m=1}^{\mathrm{N}_{\mathrm{c}}} i_{s m}(x)
$$

Where, $\mathrm{N}_{\mathrm{c}}$ is the number of circuits connected to the busbar (i.e. the number of CTs in busbar differential protection). $i_{d}(x)$ is the sampled differential current waveform. $i_{s m}(x)$ is the sampled secondary current waveform for each CT circuit $m$ (where, $m=1$ to $\mathrm{N}_{\mathrm{c}}$ ).

Each restraining current sample is the summation of the absolute values of the current samples from each CT.

$$
i_{b}(x)=\sum_{m=1}^{N_{c}}\left|i_{s m}(x)\right|
$$

Where, $i_{b}(x)$ is the sampled biasing current waveform. The magnitudes of the fundamental components of $i_{d}(x)$ and $i_{b}(x), I_{d}$ and $I_{b}$, are calculated at every sample by processing the sequential and samples using the fundamental component of a full cycle discrete Fourier transform (DFT). The operating characteristic of the relay during section $\mathrm{AB}$ is described by

$$
I_{d}>I_{\text {pick up }}
$$

The operating characteristic of the relay for each slope during section $\mathrm{BC}$ as shown in figure 1 is described by

$$
I_{d}>k I_{b}
$$


Where, $k$ is the slope of each operating characteristic of the differential relay. In the following section, the author suggests a new technique to construct similar relay characteristics but improved by making it depends on the CT saturation degrees using the results correlation as mention before.

\section{CT SATURATION DEGREES MEASUREMENTS}

The correlation coefficient between the secondary current $i_{s}(x)$ of the saturated current transformer for the faulted circuit and the summation of the secondary currents $i_{\text {sc }}(x)$ of other CT's for the unfaulted circuits is calculated in order to classify the saturation severity.

If we take a sample consisting of $N_{s}$ pairs of values $i_{s}(x)$ and $i_{s c}(x)$, we shall characterize them with the empirical correlation coefficient $(r)$. The formula for determining an approximation to the correlation coefficient $(r)$ is shown in the following equation [8]:

$$
r=\frac{\sum_{x=1}^{N_{s}} i_{s}(x) i_{s c}(x)-N_{s} \bar{i}_{s} \bar{i}_{s c}}{\sqrt{\left(\sum_{x=1}^{N_{s}} i_{s}^{2}(x)-N_{s}\left(\overline{i_{s}}\right)^{2}\right)\left(\sum_{x=1}^{N_{s}} i_{s c}^{2}(x)-N_{s}\left(\bar{i}_{s c}\right)^{2}\right)}}
$$

Where, $N_{s}=200$ (i.e., the number of samples per cycle used in the simulation).

$i_{s}(x) \quad$ is the sampled saturated secondary current of the faulted circuit.

$i_{s c}(x)$ is the summation of the un-faulted secondary current waveforms.

$$
\begin{aligned}
& \bar{i}_{s} \text { is the arithmetic mean of } i_{s}(x) . \\
& \bar{i}_{s}=\frac{1}{N{ }_{s}} \sum_{x=1}^{N}{ }_{i_{s}}(x) \\
& \bar{i}_{s c} \quad \text { is the arithmetic mean of } i_{\mathrm{sc}}(\mathrm{x}) . \\
& \bar{i}_{s c}=\frac{1}{N_{s}} \sum_{x=1}^{N} i_{s c}(x)
\end{aligned}
$$

$\mathrm{r} \quad$ is the empirical correlation coefficient of $i_{s}$ $(x)$, and $i_{s c}(x)$.

In general, the correlation coefficient measures the similarity between two signals and has a value that lies between 0 and 1 . It is used to evaluate the degree of correlating the two current waveforms, $i_{s}(x)$ and $i_{s c}(x)$. So it is considered an indication for the degree of saturation severity in the distorted secondary current signal. The lower value $\mathbf{r}$ means greater degree of saturation severity. The proposed technique uses this factor to make an adaptive busbar differential relays in order to avoid saturation effects.

\section{THE PROPOSED SCHEME}

The proposed scheme flow chart is shown by figure 2 which is discussed as follows:

\subsection{Stage 1: Fault and CT saturation detection algorithms}

In order to achieve the technique, the fault must be detected and the saturated point on current wave must be defined. Fortunately, the latter have been detected using the first derivative and developed by [9].

\subsection{Stage 2: External and internal faults discrimination}

The author suggests a method to distinguish between external and internal faults based on the fact that, following the inception of an external fault, there is a period of low differential current $\left(\mathrm{I}_{\mathrm{d}}\right)$, while all the CTs remain unsaturated, that is followed by a period of large $I_{d}$ when one of the busbar CTs enters the saturated region (the CT connected to the faulted feeder). The differential current is used to discriminate between the external and internal faults. If the magnitude of the differential quantity $i_{d}(x)$ during the interval following the fault occurrence and before the CT saturates is larger than a certain predefined threshold value, an internal fault is detected. Otherwise the fault is detected as an external. The external fault may enter saturation or not. External fault with saturation is defined as the $I_{d}$ exceeds the predetermined threshold limit after a delay time $\left(t_{d}\right)$ of the fault occurrence.

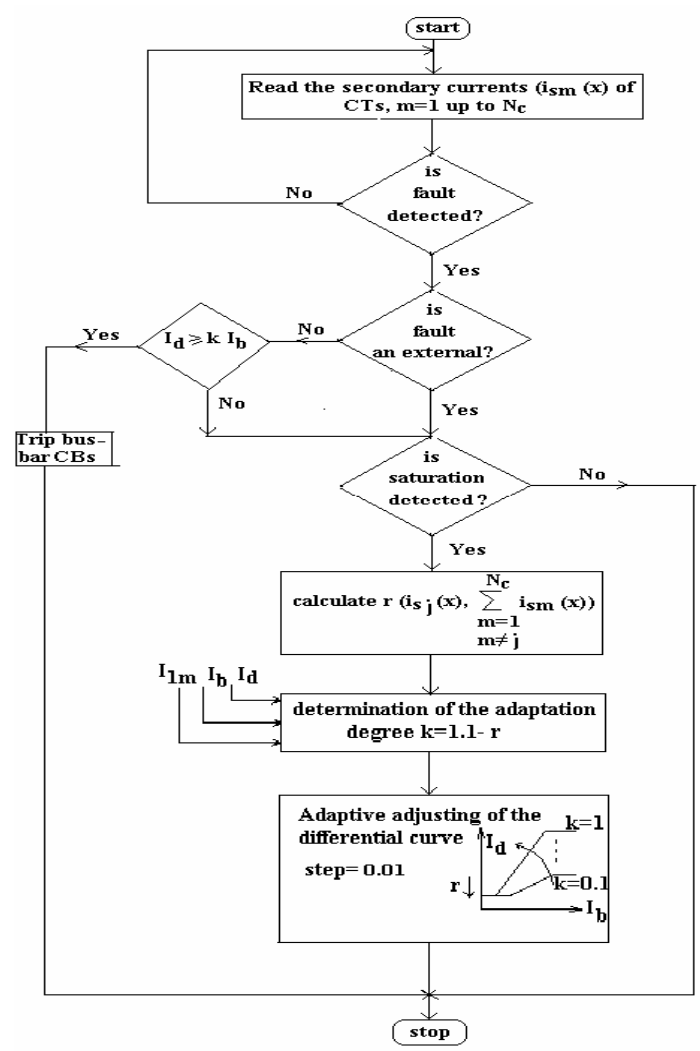

Fig. 2 Block scheme of the adaptive differential protection 


\subsection{Stage 3: Adaptive relay characteristics}

The selection of operating characteristic diagrams depends on the correlation coefficient $(r)$. So the biased differential relaying setting is adaptive setting relay based on the saturation degree. The greater the degree of saturation, the lower of the correlation coefficient (r). In such condition the stabilizing region must be increased. In other words, the slope $k$ of the relay characteristic must be increased. The author suggests using one of the two following equations:

Equation (1):

$$
k \uparrow \propto \frac{1}{r \downarrow}
$$

By using equation (6), the technique needs to use look up table for $k$ and $r$-values. Look up table determines the most suitable operating characteristics for each $r$.

Equation (2):

$$
k \uparrow=a+b \times r \downarrow
$$

Equation (7) is used as alternative to equation 6 , where $a$ and $b$ are constants.

Assuming that the relay characteristic with $k$ varies from 0.1 to 1.0 [7]. It is often more convenient to select the operating characteristic $k=1$ for saturation degree $\boldsymbol{r}=0.1$ (because the CT secondary current does not saturate suddenly) and the operating characteristic $k=0.1$ is suitable for saturation degree $r=1$. By substituting these data into eqn. 7, constants $a$ and $b$ are determined as 1.1 and -1 , respectively as described in equation 8 .

$$
k=1.1-r
$$

Now, the adaptation process can take place by applying equation 8 . This is done on line and the operating characteristic curve is obtained according to the value of correlation coefficient (r); (see Fig. 3).

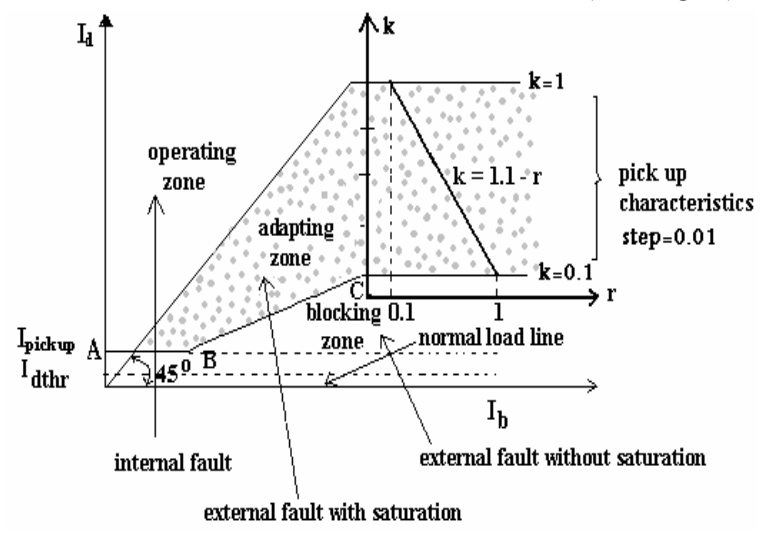

Fig. 3 operating characteristics of adaptive busbar differential protection based on CT saturation degrees

\section{TECHNIQUE EVALUATION USING SIMULATION RESULTS}

In order to examine the suggested technique under different conditions, a wide range of test cases were simulated and analyzed. The fault current waveforms were obtained from "ATP" simulation based on realistic transmission network parameters, representing various fault conditions in terms of short-circuit levels. A sampling frequency of $10 \mathrm{k} \mathrm{Hz}$ (i.e. 200 sample/cycle) is used in this study. The procedure of adaptive busbar differential protection algorithm is implemented in the "Visual basic form". The studied power system configuration is shown by figure 4 .

Test cases were simulated in the event of external fault condition at point $F_{2}$ of test network (as shown in Fig. 4). A pure resistive CT burden $\left(R_{b}\right)$ is assumed in test cases. Data used in the simulated power system is shown in figure 4 . The response results of each test case are presented with four current waveforms: the secondary fault current waveform $i_{s m}(x)$ of each CT on feeder $\mathrm{m}$, which is simulated using "ATP" under given fault conditions (the simulated saturated secondary fault current waveform $i_{s 3}(x)$ is partially distorted fault current waveform due to CT saturation effects); the summation of un-faulted secondary currents $i_{s c}(x)$ at busbar station, which is a reconstructed version of the saturated secondary current waveform; the differential current waveform $i_{d}(x)$, which is generated in the event of external faults due to the saturation condition; and The restraining current waveform $i_{b}(x)$, which is generated in such cases.

\subsection{Response of adaptive busbar differential protection}

Testing of the developed adaptive procedure for busbar differential protection with ATP simulated proved much better relay performance when compared to traditional relay version without adaptation (operating with characteristic slope $\mathrm{k}=$ $0.5)$. The results indicate that the technique is very effective in preventing false tripping during external fault conditions due to $\mathrm{CT}$ saturation (with correlation coefficient $r=0.1$ ) by selecting the more suitable operating characteristics for differential relays (the adjusted characteristic slope $\mathrm{k}=1$ ). Fig. 5 shows the response of busbar differential protection for traditional relay without adaptation (operating with characteristic slope $k=0.5$ ). Fig. 6 shows the response of busbar differential protection with adaptation (operating with characteristic slope $\mathrm{k}=1$ ). It is clear evident that the application of the adaptation_idea is useful to assure proper operation of the protection for external faults with CT saturation. 
R. Abd Allah, S. Moussa, E. H. Shehab-Eldin, M. N. G. Hamed, "Adaptive Busbar Differential Protection ..."

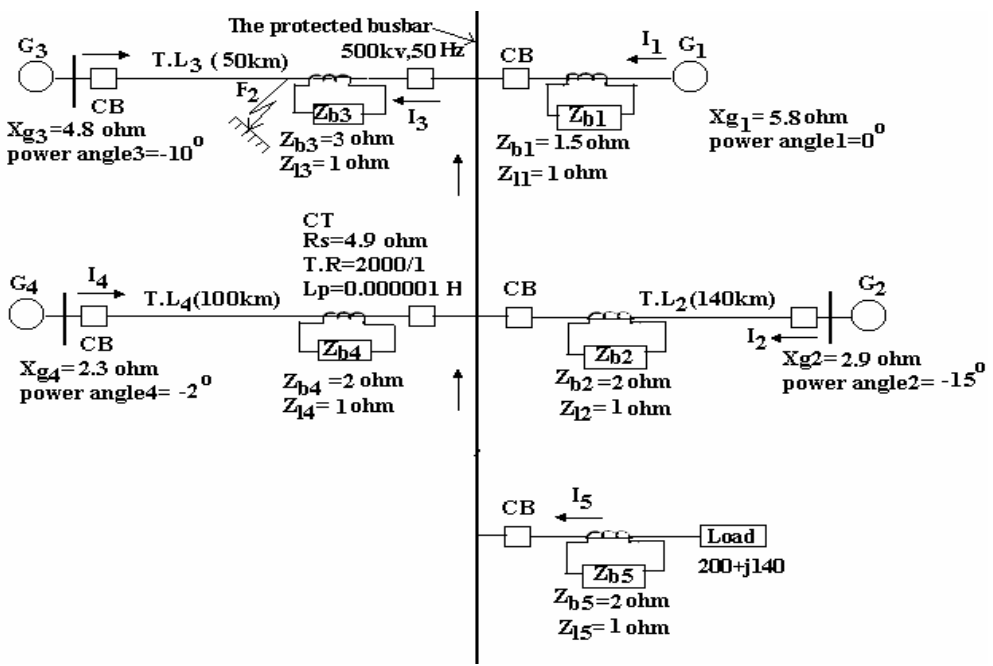

Fig.4 Single line diagram for the studied power system In case of external fault at point $\mathrm{F}_{2}$
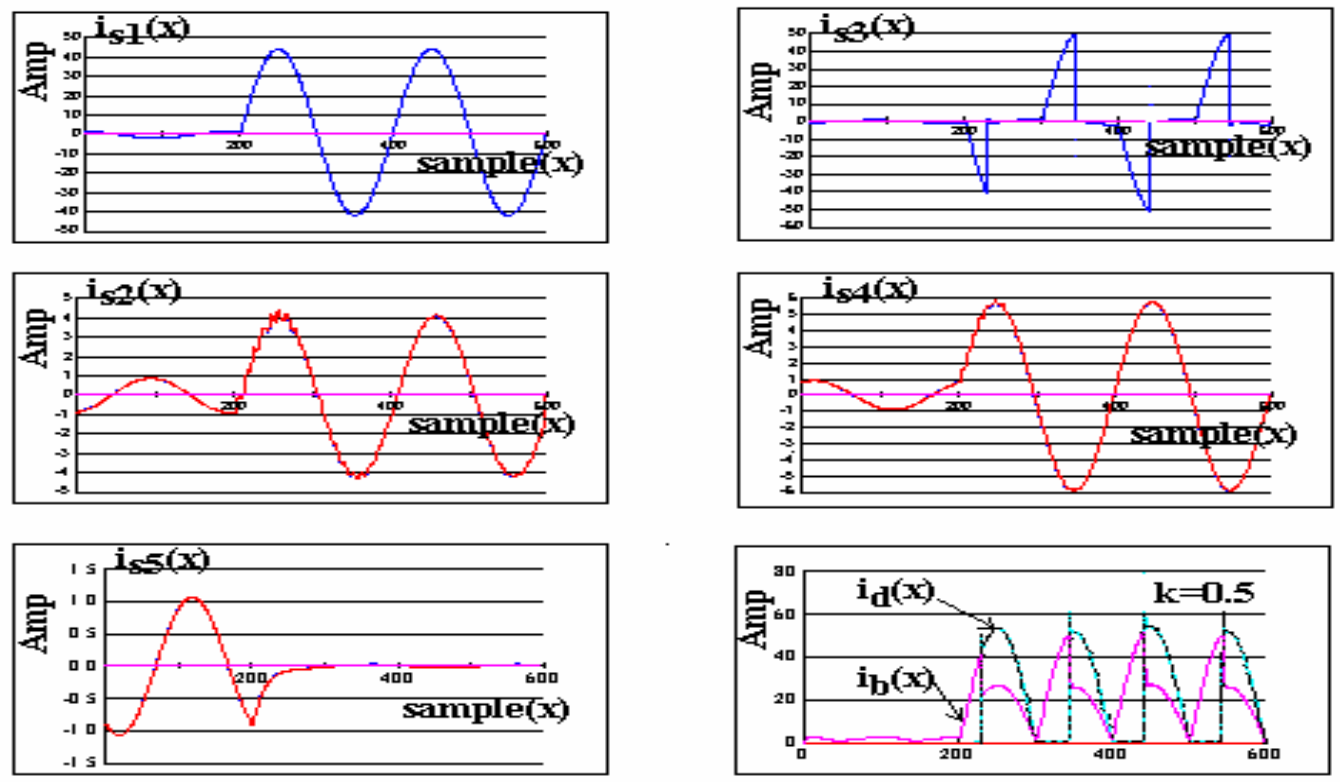

Fig.5 Response of busbar differentia Protection for traditional relay without adaptation during external fault
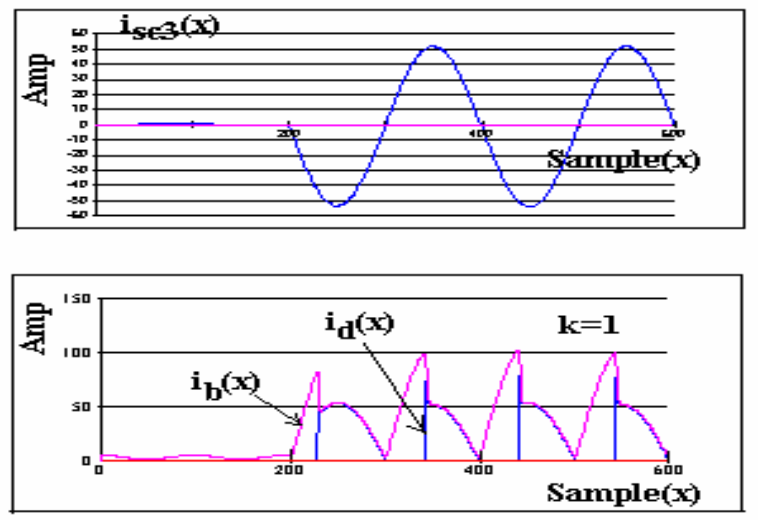

Fig.6 Response of busbar differential protection with adaptation during external fault 


\section{CONCLUSION}

This paper presents an algorithm to develop an adaptive busbar differential protection avoiding the effects of CT saturation. A correlation technique is used to measure the similarity between the saturated secondary current of a faulted-feeder and the summation of the other CTs secondary current signals at busbar station. The correlation factor is introduced and successfully implemented to adapt the relay characteristics.

The adaptive procedure developed includes appropriate on line modifying of the relay differential curve, thus enabling improvement of protection stabilization for the cases of close external faults accompanied with DC current of high time constant. Testing of the developed adaptive procedure with ATP simulated and generated current signals proved much better relay performance when compared to traditional relay version without adaptation. The results indicate that the technique is very effective in preventing false tripping during external fault conditions due to CT saturation by selecting the more suitable operating characteristics for differential relays.

\section{REFERENCES}

[1] D.A. Bradley, C.B.Gray, D.O'Kelly, “Transient compensation of current transformers", IEEE Transactions on Power Apparatus and Systems, Vol. PAS-97, no.4, July/Aug 1978.

[2] Y.C. Kang, J.K.Park, S.H.Kang, A.T. Johns, R.K. Aggarawal, "An algorithm for compensating secondary currents of current transformers", IEEE Transactions on Power Delivery, Vol.12, no.1, January 1997.
[3] D.C.Yu,Z.Wang, J.C. Cummins,H.-J. Yoon,L.A.Kojovic, and D.Stone, "Neural network for current transformer saturation correction", in proc. IEEE Transm. Distrib. Conf., New Orrleans,LA,Apr.1999.

[4] M.E. Masoud, E.H.Shehab-Eldin, M.M Eissa, and M.F.Elnagar, "A New Compensating secondary current technique for saturated current transformers", the 8thInternational Middle- East power system conference, "MEPECON 2001", PP549-555.

[5] M.A. Salem, M.I. Gilany,Z. Osman and E. aboul Zahab, "A new algorithm for compensating the secondary current during current transformer saturation", The tenth International Middle- East power systems conference "MEPECON 2005" PP 427-433.

[6] Waldemar Rebizant, Tammam Hayder, and Ludwig Schiel, "Prediction of CT Saturation Period for Differential Relay Adaptation Purposes", International-Conference-onAdvanced-Power-System-Autom, 2004.

[7] Manufacture's Catalogue of the Siprotec "Siprotec Numerical Protection Relays", Siemens AG, 2006.

[8] W. Hauschild, and W. Mosch, "Statistical Techniques for High Voltage Engineering", English edition published by peter pere grinus Ltd., London, United Kingdom 1992.

[9] R.Abd Allah, S.Moussa, E.H.Shehab-Eldin and M.N.G.Hamed, "Advanced Detection and Compensation Scheme for Current Transformer Saturation", Paper No. 134 published in the Eleventh International Middle East Power Systems Conference (MEPCON' 2006) 19-21 December 2006. 